\title{
The importance of quantitative measurement methods for uveitis: laser flare photometry endorsed in Europe while neglected in Japan where the technology measuring quantitatively intraocular inflammation was developed
}

\author{
Carl P. Herbort · Ilknur Tugal-Tutkun
}

Received: 10 March 2016/Accepted: 6 May 2016/Published online: 12 May 2016

(C) Springer Science+Business Media Dordrecht 2016

\begin{abstract}
Laser flare photometry (LFP) is an objective and quantitative method to measure intraocular inflammation. The LFP technology was developed in Japan and has been commercially available since 1990. The aim of this work was to review the application of LFP in uveitis practice in Europe compared to Japan where the technology was born. We reviewed PubMed articles published on LFP and uveitis. Although LFP has been largely integrated in routine uveitis practice in Europe, it has been comparatively neglected in Japan and still has not received FDA approval in the USA. As LFP is the only method that provides a precise measure of intraocular inflammation, it should be used as a gold standard in uveitis centres worldwide.
\end{abstract}

\section{P. Herbort}

Inflammatory and Retinal Eye Diseases, Centre for Ophthalmic Specialized Care, Montchoisi Teaching

Centre, Lausanne, Switzerland

C. P. Herbort

Department of Ophthalmology, University of Lausanne, Lausanne, Switzerland

I. Tugal-Tutkun $(\bowtie)$

Department of Ophthalmology, Istanbul Faculty of Medicine, Istanbul University, Istanbul Tip Fakultesi, Goz Hastaliklari A.D. Capa, 34093 Istanbul, Turkey

e-mail: itutkun@istanbul.edu.tr;

itutkun@yahoo.com
Keywords Laser flare photometry - Uveitis . Intraocular inflammation

In recent years, tremendous progress has been achieved in quantifying intraocular inflammatory parameters, one of them being laser flare photometry (LFP). Every effort should be done so that objective and quantitative methods replace subjective and obsolete evaluations of inflammation in uveitis practice and uveitis studies. In 1987, one of the authors was present at Tokyo University when there was a lot of activity around the development of a technology that would enable clinicians to measure objectively and quantitatively the level of intraocular inflammation. By optically determining the amount of backscattered light coming from proteins (and cells) in the aqueous produced by a laser beam directed into the anterior chamber, it would be possible, for the first time, to precisely and objectively not only to determine the exact level of intraocular inflammation but also to detect minute changes of flare during follow-up of inflammation. In 1988, the combined efforts of researchers from the Department of Ophthalmology of the University of Tokyo and engineers from the Electronics \& Optics Division of Kowa Company Ltd, Tokyo were crowned with success and were published by Sawa et al. in an article that came out in the Japanese Journal of Ophthalmology entitled "New quantitative method to determine precise concentration of proteins and cell 
number in aqueous in vivo" [1]. Initially, LFP was put forward principally as a research tool for pharmaceutical studies on intraocular inflammation [1].

Once the technology was commercially available in 1990, following the work we and others performed, it became quickly evident that LFP would prove most useful in the management of uveitis patients [2]. The most significant change LFP brought to the appraisal of uveitis was to transform flare, up to then a qualitative and subjective parameter with high intraobserver and inter-observer variance and a limited qualitative grading value from 0 to 4 , into the first, precise, quantitative and objective measurement mode of intraocular inflammation in uveitis. Due to the fact that flare was now quantifiable, flare replaced aqueous cells used until then to evaluate inflammatory activity. After several articles published in American journals, the method was, however, never integrated in uveitis practice in the USA for obscure and unexplained reasons [3-5]. In Europe as well as in other geographic areas like China, Tunisia and Turkey, the technology, little by little, became part of the uveitis care in many centres with more than 100 centres equipped with the technology in Europe. One area where LFP was adopted extensively was Turkey. This was mostly due to the leading role of the largest uveitis referral centre in Turkey (I.U., Istanbul Faculty of Medicine, Department of Ophthalmology) where the Kowa FC-2000 was started to be used on a routine basis in 2005 . Publications, as well as teaching courses, and lectures (by ITT) on the use of LFP in uveitis practice have increased the awareness of Turkish uveitis specialists, and consequently, an increasing number of referral centres have acquired the technology. This may be regarded as a major progress considering especially the proven role of LFP in monitoring disease activity in Behçet's uveitis and the high prevalence of the disease in Turkey.

It was assumed, naturally, that the technology was also widely used in Japan who had had an early access to LFP. It was a considerable surprise when one of the authors realized that this did not seem to be the case. He came across an article where inflammation was evaluated in uveitis cases before and after vitrectomy where the outcome relied only on clinical signs and outcomes, not including quantitative LFP data, although LFP would have brought the needed accuracy and quantification to prove a significant difference among some of the groups that was not obtained using poorly discriminative clinical outcomes [6]. Further to this finding, when checking on PubMed from which area articles published on LFP and uveitis were coming from, relatively few articles came from Japan. Indeed, if we except the articles published in 1988 and 1989 on the technology itself and if we only consider articles on applications of the method, the number of articles published on LFP and uveitis and even on LFP and inflammation coming from Japan was astonishingly low in the international literature. Roughly, the number of articles on LFP or where LFP use was cited as the measurement method of intraocular inflammation was 89/143 for Europe, 17/143 for China with a frequency increasing over the years and an expected low proportion of $9 / 143$ for the USA. Comparatively, the proportion of articles coming from Japan was relatively low and decreasing over the years (17/143), indicating that the use of LFP for uveitis seemed to be somewhat neglected. This impression was further confirmed when discussing with colleagues working in some major uveitis centres in Japan that did not seem to use LFP technology.

The aim of this work was to try to elucidate the reason why a method that today can be considered the gold standard for the measurement of intraocular inflammation has been neglected in the country where the technology was born.

At first, if we follow the indications of the original article published by Sawa et al. [1], LFP was destined especially to be used for experimental and clinical studies on inflammation and indeed it was used in innumerable such studies. It permitted to compare the anti-inflammatory effect of various topical and systemic agents. After the availability of LFP, it is not thinkable any more to perform studies testing the effect of anti-inflammatory substances without using the quantitative technology represented by LFP.

However, it is in everyday uveitis practice that LFP technology has proven most useful. LFP-measured flare has become the only quantifiable parameter for most cases of intraocular inflammation both in anterior and posterior segment inflammation. In posterior uveitis the method proved useful for the follow-up of inflammation, as long as there was a minimum of associated anterior chamber spill-over flare of 15 photon counts per millisecond [4]. It was shown that LFP was not only essential in the assessment and the follow-up of acute inflammation but also in chronic disruption of the blood-aqueous barrier. Even in the 
absence of cells, LFP could detect active and treatable inflammation as was shown in a group of patients with JIA-associated uveitis without aqueous cells, where a significant flare reduction after introduction of maximal therapy was obtained [7]. It was further shown that LFP-flare was superior to slit-lamp cell evaluation for the follow-up of inflammation in acute inflammation measured in HLA-B27-related uveitis [8].

Indeed, it has to be realized that LFP fundamentally changed the approach to intraocular inflammation in uveitis. The availability of such a quantitative and objective parameter giving reproducible results hence should become the preponderant parameter to measure and follow intraocular inflammation. The rule prevailing before LFP, saying that, in the absence of cells in the aqueous, there was no active inflammation, had to be revised. Especially in uveitis related to juvenile idiopathic arthritis (JIA), chronic evolution can be associated with very high flare values indicating massive blood-ocular barrier (BOB) disruption not always associated with the presence of aqueous cells. Very often these cases are under minimal treatment because few or no cells are present. If maximal treatment is applied to such cases, a substantial reduction of flare occurs, only quantifiable with LFP, showing that an important part of what was thought to be an irreversible BOB disruption can be reversed leading to functional improvement. In such situations, LFP was of crucial importance to adjust management. This is only one example illustrating the substantial gain of sensitivity brought by LFP in the management of uveitis [7].

In essence, LFP is to uveitis what applanation tonometry is to glaucoma, and allows to measure at any given time-point the level of intraocular inflammation. The progress of LFP over slit-lamp evaluation of anterior chamber inflammation for uveitis could be compared to the progress brought about by applanation tonometry over digital/finger evaluation of intraocular pressure for glaucoma, replacing a qualitative and subjective measurement method by a quantitative and objective one. Of course, no glaucoma centre would consider to follow intraocular pressure by such an inadequate and qualitative method such as finger/digital evaluation of intraocular pressure. Accuracy and reproducibility of applanation tonometry are not questioned anymore, whereas ophthalmologists may still be concerned about intra- and inter-observer variability of LFP measurements. Several studies conducted in the 1990s have shown the reproducibility and validity of LFP measurements [913]. To avoid the introduction of bias in flare measurements, five to seven sequential measurements are taken, readings with background scatter less than $15 \%$ are averaged, and the standard deviation of the acceptable readings is calculated automatically. Coefficient of variation is less than $10 \%$ at repeated measurements by the same technician or by different technicians. The whole measurement process does not take more than a few minutes and does not cause any discomfort to the patient, at least not as much as applanation tonometry or pneumotonometry. There are limitations, however, such as the presence of central corneal opacity or a very shallow anterior chamber, where no measurement can be obtained. Furthermore, reliable measurements may not be obtained in eyes with severe keratic precipitates, extensive posterior synechiae or mature cataract due to increased background scattering of light. Such limitations exist for any other technology that we routinely use, such as automatic refractometer or optical coherence tomography, both of which cannot be reliably used in eyes with significant media opacity. Reliable flare measurements can be performed in the vast majority of patients with uveitis, and in fact, LFP readings are now taken as the gold standard to test variability of clinical grading of anterior chamber flare by clinicians with different levels of experience [14].

It was shown that LFP-flare increase or decrease could be detected before any noticeable clinical evolution could be recorded [5]. This could be noticed both in high and low flare situations. Such flare changes can absolutely not be recorded by slit-lamp evaluation of flare, and their measurement by LFP gives a tremendous security in the follow-up of uveitis cases of which the uveitis specialist should not deprive himself but which he should use as a precious help to take therapeutic decisions.

A recent study has shown that in a substantial proportion of cases, LFP was at the origin of a change of therapeutic decisions taken before the LFP-values were known to the clinician [14]. Therefore, it is difficult to understand why a centre specialized in uveitis would deprive itself of such a quantitative method to help uveitis management. It is also expected from leading uveitis centres to perform clinical studies on inflammation that nowadays can hardly be 
submitted without reporting quantitative data available thanks to LFP.

Some reasons can be found to explain why LFP has never been integrated into routine uveitis practice in uveitis centres in the USA. The device has never been FDA approved for unknown reasons, whereas it received immediately the CE label opening its diffusion in Europe. Moreover, LFP is not reimbursed in the USA and the technology has been generated outside the USA, so probably attracting less attention and less incentive to use it. These reasons and maybe some more have led to the technology being neglected in the USA. It was also excluded from the parameters put forward in the standardization of uveitis nomenclature (SUN) in favour of existing non-quantitative grading systems published more than 50 years ago $[15,16]$.

It is, however, much more difficult to understand and to find an explanation why clinicians and uveitis centres in Japan seem to have attributed limited importance to the use of LFP in uveitis and why it is so under-used in the country that saw the development of the technology. It is really an enigma why Japanese uveitis specialists did so little to promote LFP and did little to help work towards the universal recognition and use of this first quantitative parameter to quantitate intraocular inflammation. Did the Japanese uveitis specialists active at the moment when LFP was developed, not bother to integrate LFP in their daily management of uveitis cases because it was stated in the first publication that the instrument was destined only to be used for research purposes? Moreover, could it be hypothesized that Japanese uveitis specialists did not pursue the use of LFP in uveitis when not frankly neglecting it because the methodology was neither adopted nor considered in the USA and hence lead to the underestimation of its importance or to its omission altogether in Japan? Is it possible that there was no incentive to use it and publish reports on its use in uveitis, as the American peer-reviewers would not consider the technology, generating a quasi-autocensorship among Japanese clinicians and researchers? In recent work performed on inflammatory conditions such as Behçet's uveitis, pre-eminence was still given to clinical signs, indeed an important part of the evaluation, while leaving out LFP, when our Turkish colleagues showed LFP follow-up to be extremely useful to monitor inflammatory evolution in Behçet's uveitis [17-19].
The methodology not having found its way into routine practice in specialized uveitis centres in Japan when it became available, the next generation of uveitis specialists were not inclined to use the technology, not having been exposed to LFP by their teachers and mentors. Indeed, not much was done to strongly promote LFP which failed to take the place it should occupy. Today LFP and other quantitative measurement methods such as dual fluorescein and indocyanine angiography grading for posterior uveitis $[20,21]$ have a hard time to replace obsolete and inadequate parameters to measure outcomes in uveitis studies imposed by some opinion leaders.

Major uveitis centres should be equipped with LFP technology, taking into account the additional security it gives to inflammation follow-up. The technology should also be available in these centres for conducting uveitis studies. The availability of LFP is furthermore important to give the possibility to junior colleagues to develop clinical research projects using LFP.

We can only hope that our assumptions are too pessimistic. Hopefully, the neglect of LFP for uveitis in Japan is not as generalized as it seems from the scarcity of international publications on the methodology and its use. Young uveitis specialists from Japan were encountered who rely on LFP and have integrated it in their clinical practice and their publications. If there is such a trend, it should find its way into the international literature with increasing reports on measurement of intraocular inflammation using LFP in publications on uveitis cases and uveitis studies [22].

\section{References}

1. Sawa M, Tsurimaki Y, Tsuru T, Shimizu H (1988) New quantitative method to determine protein concentration and cell number in aqueous in vivo. Jpn $\mathrm{J}$ Ophthalmol 32:132-142

2. Tugal-Tutkun I, Herbort CP (2010) Laser flare photometry: a noninvasive, objective and quantitative method to measure intraocular inflammation. Int Ophthalmol 30:453-464

3. Herbort CP, Guex-Crosier Y, de Ancos E, Pittet N (1997) Use of laser flare photometry (LFP) to assess and monitor inflammation in uveitis. Ophthalmology 104:64-72

4. Guex-Crosier Y, Pittet N, Herbort CP (1994) Evaluation of laser flare-cell photometry in the appraisal and management of intraocular inflammation in uveitis. Ophthalmology 101:728-735 
5. Guex-Crosier Y, Pittet N, Herbort CP (1995) Sensitivity of laser flare photometry to monitor inflammation in uveitis of the posterior segment. Ophthalmology 102:613-621

6. Takayama K, Tanaka A, Ishikawa S, Mochizuki M, Takeuchi M (2016) Comparison between outcomes of vitrectomy in granulomatous and nongranulomatous uveitis. Ophthalmologica 235:18-25

7. Herbort CP (2009) Laser flare photometry. Chapter 3. In: Gupta A, Gupta V, Herbort CP, Khairallah M (eds) Uveitis text and imaging. Jaypee, New Delhi, pp 28-49

8. Bernasconi O, Papadia M, Herbort CP (2010) Sensitivity of laser flare photometry compared to slit-lamp cell evaluation to monitor anterior chamber inflammation in uveitis. Int Ophthalmol 30:495-500

9. Shah SM, Spalton DJ, Smith SE (1991) Measurement of aqueous cells and flare in normal eyes. $\mathrm{Br} \mathrm{J}$ Ophthalmol 75:348-352

10. Shah SM, Spalton DJ, Taylor JC (1992) Correlations between laser flare measurements and anterior chamber protein concentrations. Invest Ophthalmol Vis Sci 33:2878-2884

11. El-Maghraby A, Marzouki A, Matheen TM, Souchek J, Van Der Karr M (1992) Reproducibility and validity of laser flare/cell meter measurement as an objective method of assessing intraocular inflammation. Arch Ophthalmol 110:960-962

12. Rodinger ML, Hessemer V, Schmitt K, Schickel B (1993) Reproducibility of in vivo determination of protein and particle concentration with the laser flare-cell photometer. Ophthalmologe 90:742-745

13. el-Harazi SM, Feldman RM, Chuang AZ, Ruiz RS, Villanueva G (1998) Reproducibility of the laser flare meter and laser cell counter in assessing anterior chamber inflammation following cataract surgery. Ophthalic Surg Lasers 29:380-384

14. Agrawal R, Keane PA, Singh J, Saihan Z, Kontos A, Pavésio CE (2016) Comparative analysis of anterior chamber grading between clinicians with different levels of experience and laser flare photometry. Ocul Immunol Inflamm 24:184-193

15. Jabs DA, Nussenblatt RB, Rosenbaum JT (2005) Standardization of uveitis nomenclature for reporting clinical data. Results of the first international workshop. Am J Ophthalmol 140:509-516

16. Hogan MJ, Kimura SJ, Thygeson P (1959) Signs and symptoms of uveitis. 1. Anterior uveitis. Am J Ophtalmol 47:155-170

17. Tanaka R, Murata H, Takamoto M, Ohtomo K, Okinaga K, Yoshida A, Kawashima H, Nakahara H, Fujino Y, Kaburaki $\mathrm{T}$ (2015) Behçet's disease ocular attack score 24 and visual outcome in patients with Behçet's disease. Br J Ophthalmol. doi:10.1136/bjophthalmol-2015-307362

18. Tugal-Tutkun I, Cingü K, Kir N, Yeniad B, Urgancioglu M, Gül A (2008) Use of laser flare-cell photometry to quantify intraocular inflammation in patients with Behçet uveitis. Graefes Arch Clin Exp Ophthalmol 246:1169-1177

19. Yalcindag FN, Kiziltunc PB, Savku E (2016) Evaluation of intraocular inflammation with laser flare photometry in Behçet uveitis. Ocul Immunol Inflamm 30:1-5. doi:10. 3109/09273948.2015.1108444

20. Tugal-Tutkun I, Herbort CP, Khairallah M, Angiography Scoring for Uveitis Working Group (ASUWOG) (2010) Scoring of dual fluorescein and ICG inflammatory angiographic signs for the grading of posterior segment inflammation (dual fluorescein and ICG angiographic scoring system for uveitis). Int Ophthalmol 30:539-552

21. Tugal-Tutkun I, Herbort CP, Khairallah M, Mantovani A (2010) Interobserver agreement in scoring of dual fluorescein and ICG inflammatory angiographic signs for the grading pf posterior segment inflammation. Ocul Immunol Inflamm 18:385-389

22. Herbort CP, Tugal-Tutkun I (2010) Laser flare (cell) photometry: 20 years already. (Editorial). Int Ophthalmol 30:445-447 